\title{
Laser Flash Photolysis Studies of Some Aliphatic Ketone Polymers and the Model Compound, 3,3-Dimethyl-2-butanone
}

\author{
I. NAITO* and W. SCHNABEL \\ Hahn-Meitner-Institut für Kernforschung, Bereich Strahlenchemie, \\ Glienicker Str. 100, D-1000 Berlin 39, \\ Federal Republic of Germany
}

(Received April 18, 1983)

\begin{abstract}
Poly(3-buten-2-one) (PMVK), poly(4,4-dimethyl-1-penten-3-one) (PBVK), and poly(3-methyl-3-buten-2-one) (PMIK) were irradiated in a dichloromethane solution with $15 \mathrm{~ns}$ flashes of $265 \mathrm{~nm}$ light. In the case of PMVK, the existence of 1,4-biradical $\left(k=2.5 \times 10^{7} \mathrm{~s}^{-1}\right)$ was shown by transient absorption measurements. In the cases of PBVK and PMIK, the absorption spectra of acyl radicals originating from $\alpha$-cleavage were detected at the end of each flash. Emission measurements yielded only prompt fluorescence. Kinetic analysis of the decay of the transient recorded with $\mathrm{PBVK}$ yielded evidence for the formation of a polymeric acyl radical

$-\mathrm{CH}_{2}-\mathrm{CH}-\mathrm{CH}_{2}-$,

. CO

which decomposed when $k=4 \times 10^{6} \mathrm{~s}^{-1}$. For PMIK and 3,3-dimethyl-2-butanone (a model compound) the absorption spectrum of acetyl radical was obtained in the range of $(1.5-2.0) \times 10^{5} \mathrm{~s}^{-1}$. KEY WORDS Laser Flash Photolysis / 1,4-Biradical / Polymeric Acyl

Radical / Acetyl Radical /
\end{abstract}

Flash photolysis studies have recently been applied increasingly to the elucidation of the mechanism of photodegradation of polymers and the kinetics of relevant elementary reactions. ${ }^{1-4}$ Because of the high time resolution of the techniques applied, evidence for the existence and reactivity of many short-lived intermediates have been obtained. In various cases it has been possible to corroborate or discard mechanistic concepts developed earlier on the basis of stationary irradiation experiments. Optical absorption and emission have been used most frequently for the detection of transient species (excited states, free radicals, and radical ions). Also, the light scattering detection has been applied in the authors' laboratory to investigate the kinetics of photo- chemical processes involving size change in macromolecules (main chain scission or crosslinking). ${ }^{5}$

The present paper reports on absorption and emission studies carried out with some aliphatic ketone polymers and a low molecular weight model compound. These studies are a continuation of former investigations dealing with aromatic ketone compounds. ${ }^{6-8}$

According to present knowledge, ${ }^{9-13}$ photochemical reactions of polymers of the structure

$$
\left[\begin{array}{l}
\mathrm{R}_{2} \\
-\mathrm{C}-\mathrm{CH}_{2}- \\
\stackrel{\mathrm{C}}{\mathrm{T}}=\mathrm{O} \\
\mathrm{R}_{1}
\end{array}\right]_{n}
$$

initiated by the excitation of $n-\pi^{*}$ transitions

* On leave from Kyushu Sangyo University, Department of Photography, Matsugadai, Higashi-ku, Fukuoka 813, Japan. 
in carbonyl groups, can proceed by the socalled Norrish Types I and II processes, as depicted in Scheme 1.

If $R_{2}$ is a substituent other than hydrogen, the Type I process is essentially responsible for the photochemical alterations of the polymer. On the other hand, Types I and II processes can occur in parallel if $R_{2}$ is hydrogen, as has been inferred from product analysis studies with aliphatic and aromatic ketones.

It appears that the Type I process may be important relative to the Type II process if the approach of the excited carbonyl groups and $\gamma$ hydrogens is sterically impeded by the presence of bulky substituents.

As far as the Type II process is concerned, the detection of 1,4-biradicals produced by the abstraction of the $\gamma$-hydrogens (see Scheme 1) has been a subject of various flash photolysis studies with both low and high molecular weight ketones. Kinetic spectrosicopical data on the aromatic carbonyl compound, poly(phenyl vinyl ketone), and data from scavenging experiments with paraquat ions obtained with $\gamma$-methylvalerophenone were considered as indicative of the 1,4-biradical lifetimes of 50 to $100 \mathrm{~ns}$ in these cases. ${ }^{3}$ Data for the aliphatic carbonyl compounds were obtained with 2-hexanone and 5-methyl-2hexanone. ${ }^{4}$

In the following sections, experiments using several aliphatic ketones are described and should provide additional information on the competition of Types I and II processes and the possibility of detecting biradicals in the Type II process as relatively long living transients.

\section{EXPERIMENTAL}

\section{(a) Materials}

The ketones used in this work are listed in Table I. PMVK was prepared by polymerization of the neat monomer by $\gamma$-irradiation as described before. ${ }^{4}$ PBVK and PMIK were obtained by radical polymerizations of the respective monomers in dioxane solutions at $323 \mathrm{~K}$ using AIBN as the initiator. 3,3Dimethyl-2-butanone was dried with anhydrous sodium sulfate and subsequently distilled twice via a splitted-tube column (Fischer, Bonn).

Dichlomethane (E. Merck, for fluorescence spectroscopy) was washed five times with aqueous sodium carbonate solution $(5 \%)$ and five times with distilled water. After drying on anhydrous calcium chloride, it was distilled twice via the splitted-tube column.

Acetonitrile (E. Merck, for spectroscopy) was refluxed five times for $10 \mathrm{~h}$, each time on $\mathrm{P}_{2} \mathrm{O}_{5}$ followed by distillation via the splitted-tube column.

Tetrahydrofuran (E. Merck, for spectroscopy) was passed through a column containing basic alumina and then distilled twice under argon from $\mathrm{KOH}$ pellets.

\section{(b) Apparatus and Irradiation of Samples}

A neodymium YAG laser was used in conjunction with two frequency doublers. ${ }^{14}$ The output of $265 \mathrm{~nm}$ light was about $2 \times 10^{-8}$ einstein per flash $(c a .10 \mathrm{~mJ})$, as determined by tetraphenylcyclobutane as the actinometer ${ }^{4}$ with $\phi($ trans -stilbene $)=0.68$. Filters from Schott (UG 5 and UG 11) were used to reduce the light intensity. Sample solutions were saturated with purified argon prior to irradiation in quartz cells.

The sample concentration, with respect to carbonyl groups, was about $3.1 \times 10^{-2}$ mol dm ${ }^{-3}$. This concentration corresponded to an optical density of about 0.5 at $\lambda=$ $265 \mathrm{~nm}$.

The emission and absorption spectra reported in this work were normalized with respect to a laser output of $1.9 \times 10^{-8}$ einsteins per flash.

\section{RESULTS AND DISCUSSION}

\section{Emission Measurements}

In all cases, a strong emission of light was 


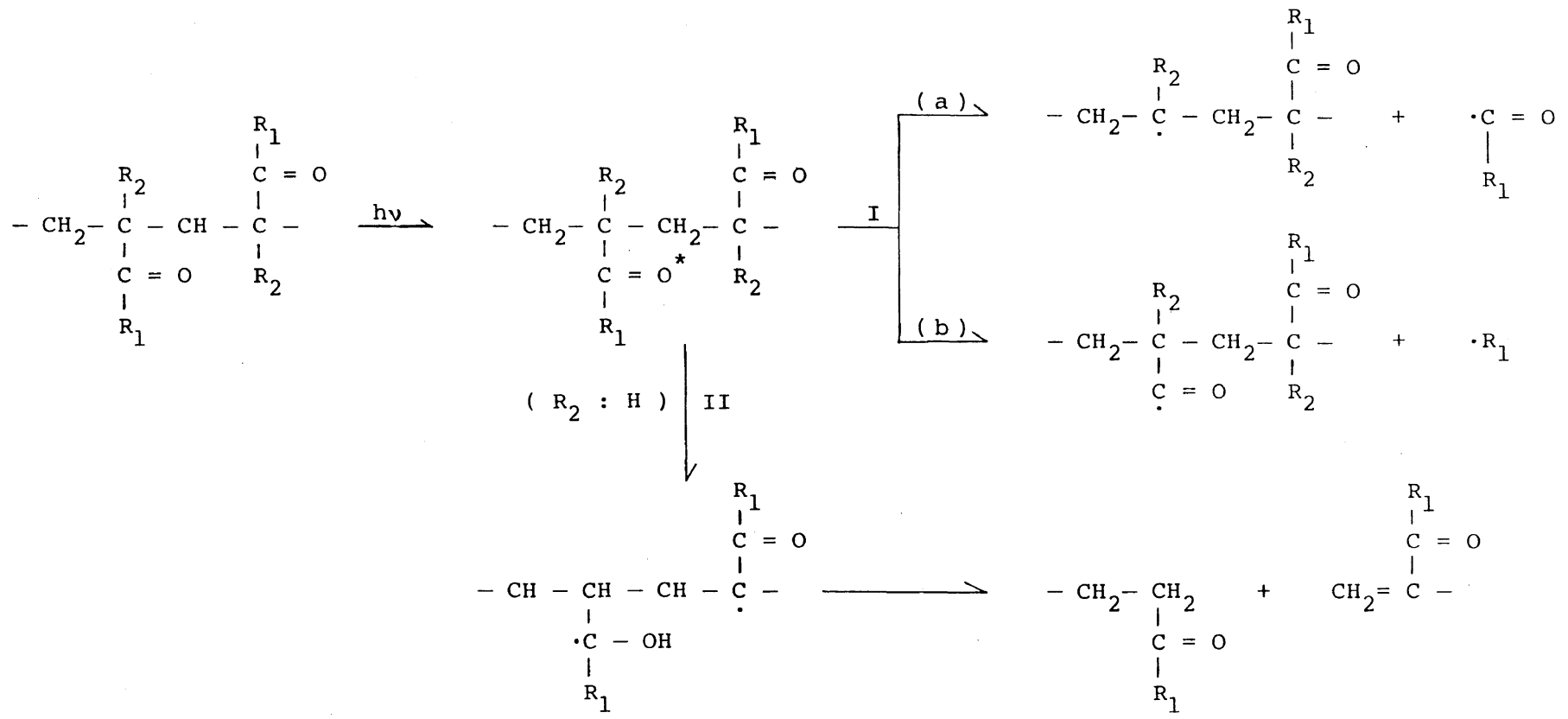

Scheme 1. Photochemical reactions in polymers following $n-\pi^{*}$ transitions in carbonyl groups. 
Table I. Ketone polymers and the model compound

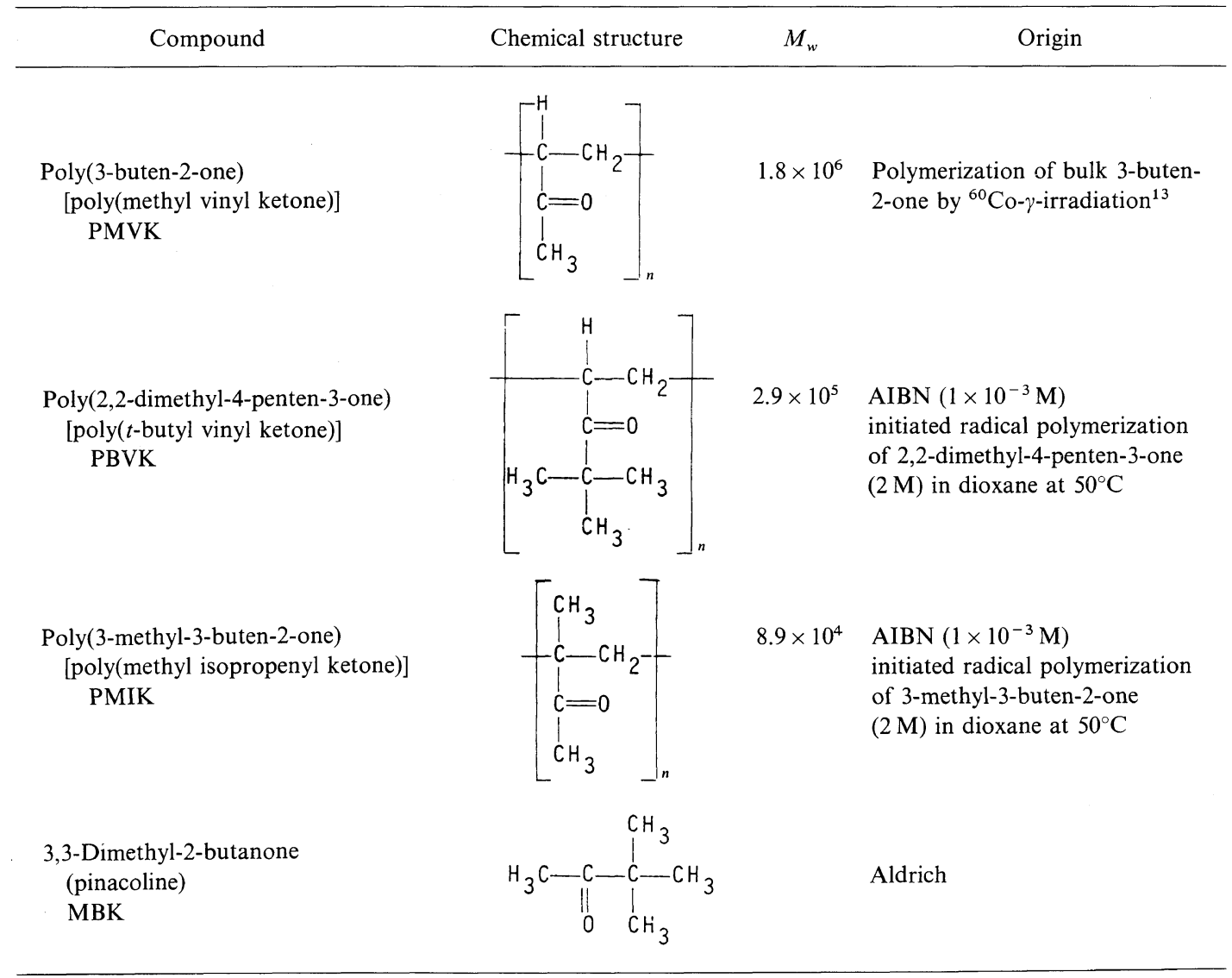

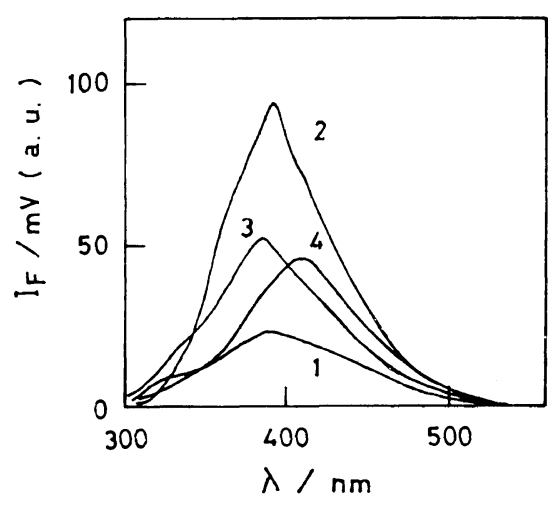

Figure 1. Emission spectra of PMVK (1), PBVK (2), PMIK (3), and MBK (4) recorded during the flash. The spectra were normalized with respect to the absorbed dose but not corrected for the sensitivity of the photomultiplier. observed upon laser flash photolysis of argonsaturated solutions at $\lambda_{\text {inc }}=265 \mathrm{~nm}$ and $293 \mathrm{~K}$. The emission was short-lived $(k>$ $\left.2 \times 10^{8} \mathrm{~s}^{-1}\right)$, i.e., the shape of the emission signal corresponded to the flash profile. The emission spectra shown in Figure 1 agree with the fluorescence spectra, reported in the literature. ${ }^{15}$ It should be pointed out that only quick and not delayed fluorescence or phosphorescence was detected. Therefore, no evidence for the triplets was obtained.

\section{Absorption Measurements}

Figure 2 shows the absorption spectra recorded with argon-saturated solutions immediately and at various periods following the flash. The spectrum obtained with 3,3- 


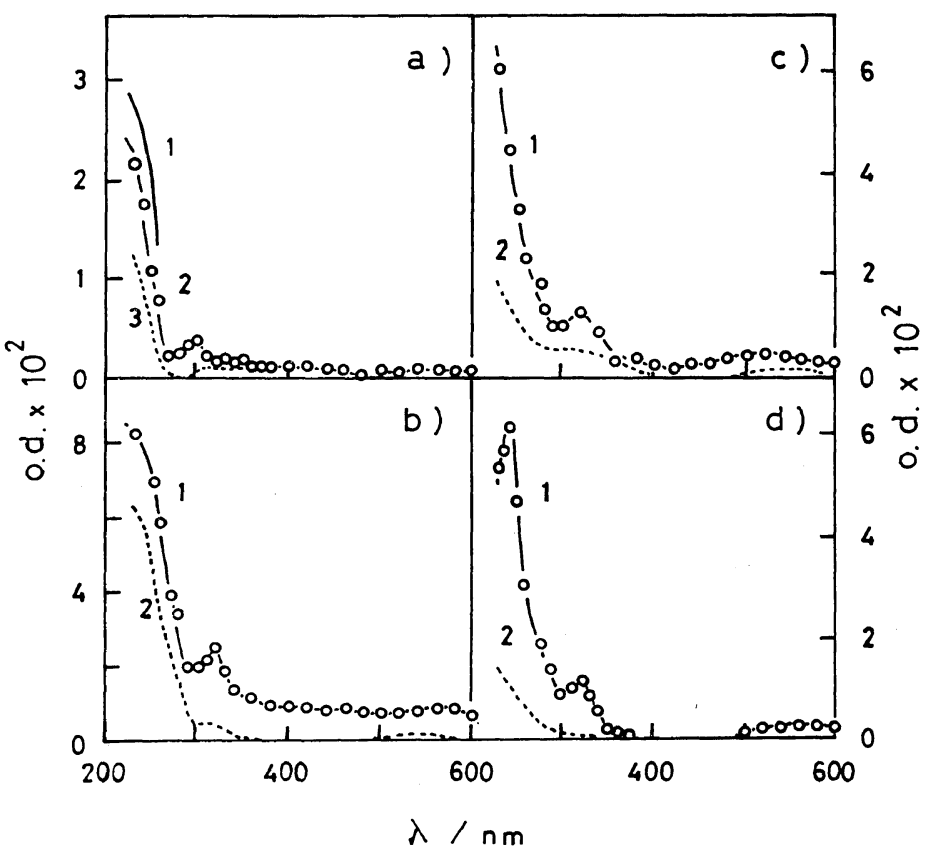

Figure 2. Transient absorption spectra of PMVK (a), PBVK (b), PMIK (c), and MBK (d) measured immediately and at various periods after the flash in dichloromethane $\left(3.2 \times 10^{-2}\right.$ basemol dm $\left.{ }^{-3}\right)$. The numbers at the curves denote the following delay times. (a): $t=0$ (1), $t=50 \mathrm{~ns}(2)$, and $t=200 \mathrm{~ns}$ (3). (b): $t=0$ (1) and $t=2 \mu \mathrm{s} \mathrm{(2).} \mathrm{(c):} t=0$ (1) and $16 \mu \mathrm{s}$ (2). (d): $t=0$ (1) and $t=7 \mu \mathrm{s} \mathrm{(2).} \mathrm{The} \mathrm{spectra} \mathrm{were} \mathrm{normalized}$ with respect to the absorbed dose.

dimethyl-2-butanone (MBK) is quite helpful for interpreting the remaining spectra shown in Figure 2. Actually, the attribution of the absorption peaks at $\lambda>300 \mathrm{~nm}$ in the spectrum (d) in Figure 2 to the acetyl radical is straightforward. The maxima observed at $320 \mathrm{~nm}$ and at about $540 \mathrm{~nm}$ correspond fairly well to the maxima values $(340,500$, and $540 \mathrm{~nm}$ ) reported by Noda et al. ${ }^{16}$ The photolysis of MBK yielded acetyl and $t$-butyl radicals with $\phi_{\alpha} 0.51,{ }^{17} 0.8^{18}$;<smiles>CC(=O)C(C)(C)C</smiles>

Alkyl radicals do not absorb light at $\lambda>300 \mathrm{~nm}$. Assignment to the triplet can be excluded, since emission measurements showed no species with a lifetime corresponding

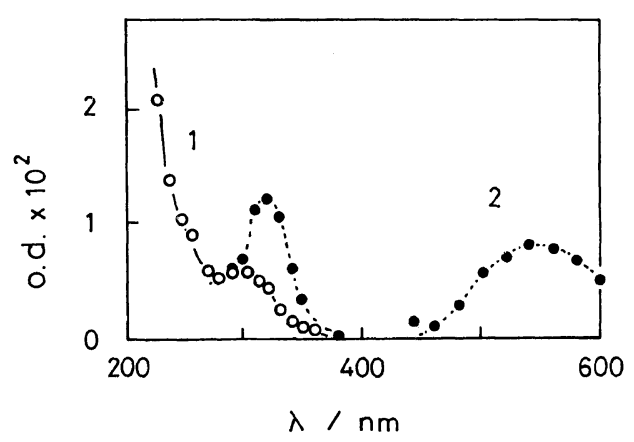

Figure 3. Difference absorption spectra. (1): PMVK, difference of spectra recorded at $t=50 \mathrm{~ns}$ and $t=200 \mathrm{~ns}$. (2): MBK, difference of spectra recorded at $t=0$ and $t=7 \mu$ s.

to that of the species having the absorption spectrum in Figure 2(d) $(\tau=5 \mu \mathrm{s})$. The spectra observed immediately after the flash with PBVK and PMIK, Figures 2(b) and 2(c), are very similar to the spectrum shown in Figure 


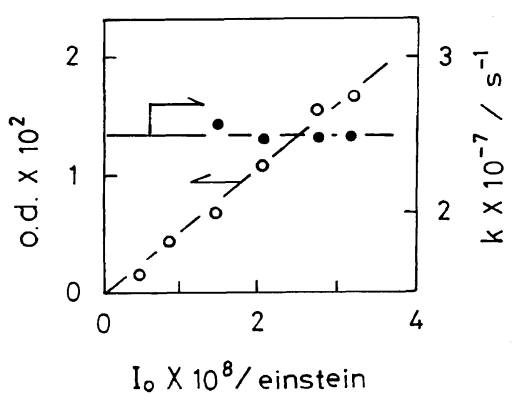

Figure 4. Flash photolysis of PMVK in dichloromethane. The initial transient absorption (left ordinate) and rate constant of the decay of the initial absorption as a function of exposure dose.

2(d) and were thus also assigned to acyl and acetyl radicals, respectively. A different situation was encountered with PMVK. In this case, the spectrum recorded initially, i.e., $50 \mathrm{~ns}$ after the start of the flash showed a weak absorption maximum at about $300 \mathrm{~nm}$ but none at $\lambda>400 \mathrm{~nm}$, as evident from Figures 2(a-2) and 3 (line 1), in which difference spectra obtained with PMVK and MBK are presented for comparison. The initial spectrum observed with PMVK was tentatively ascribed to the 1,4-biradical:<smiles>CC(=O)CC(C)O</smiles>

Assignment of this spectrum to the carbonyl triplet was discarded since the $\mathrm{T}-\mathrm{T}$ absorption spectra of aliphatic ketones such as propanone and butanone ${ }^{19}$ do not possess a maximum at about $300 \mathrm{~nm}$.

In Figure 4, the initial optical density for PMVK increased linearly with the exposure photons. The first-order decay rate constant $\left(k=2.5 \times 10^{7} \mathrm{~s}^{-1}\right)$ was independent of the quantity of photons, as also shown in Figure 4. This is in accordance with the assignments of the spectra to the 1,4-biradicals and not to the

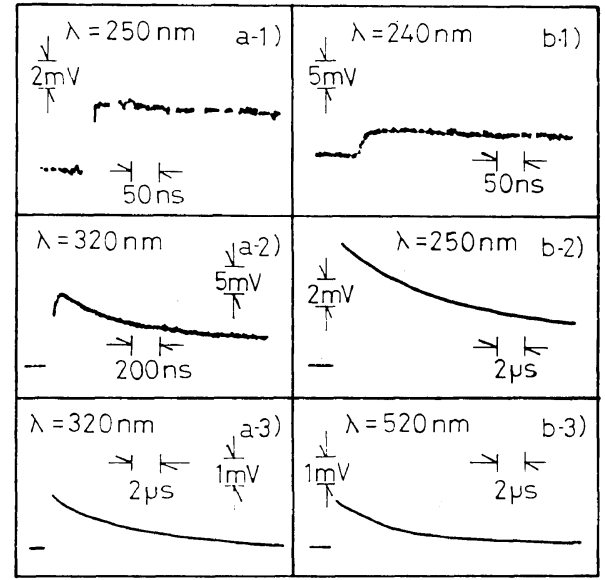

Figure 5. Oscilloscope traces illustrating the formation and decrease in transient absorption observed with PBVK (a) and PMIK (b) in argon-saturated dichloromethane solution. Exposure dose: $2-3 \times 10^{-8}$ einstein per flash.

triplets. At high doses, corresponding to high initial concentrations, the triplets probably undergo $T-T$ annihilation. The occurrence of this reaction should be indicated by secondorder kinetics or, at least, by a dependence of the experimental first-order rate constant on the absorbed photons per flash.

As can be seen from Figure 3 (line 1) the transient spectrum, recorded $200 \mathrm{~ns}$ after the start of the flash strongly resembles the spectrum of the acetyl radical, thus indicating its existence. This spectrum was rather long-lived and decayed in a $\mu$ s range with a first-order rate constant of $1.5 \times 10^{5} \mathrm{~s}^{-1}$. These findings show that, in the case of PMVK, both Types I and II processes (as depicted in Scheme 1) occur simultaneously.

With PBVK and PMIK, relatively longlived absorptions were also observed, as shown by the oscilloscope traces in Figure 5. The decay of these absorptions did not follow simple kinetic laws. Thus, it was concluded that the observed spectra consisted of the spectra of different radicals decomposing at different rates. Scheme 1 shows that the $\alpha$-scission produces macroradicals along with low mo- 
lecular weight radicals. It is possibly the absorption of macroradicals which overlaps the absorption of (low molecular weight) acyl radicals in the cases of PBVK and PMIK, respectively. From Scheme 1, it is obvious that Type I reactions can proceed via two routes. Both routes may be important, particularly in the case of PBVK. The polymeric acyl radicals of the type $\mathrm{R}-\mathrm{C}=\mathrm{O}$, generated during the $\alpha$ scission of PBVK according to route (b), probably possess an absorption spectrum similar to that of the acetyl radical $\left(\mathrm{H}_{3} \mathrm{C}-\mathrm{C}=\mathrm{O}\right)$. However, they might decompose more rapidly according to reaction (2):

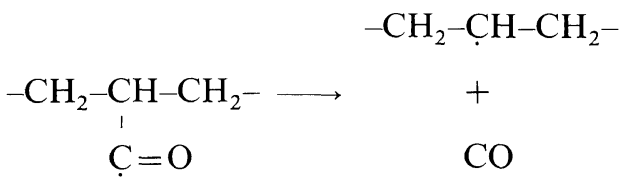

A rate constant of about $4 \times 10^{6} \mathrm{~s}^{-1}$ was estimated for reaction (2) on assuming that a more rapid decay of the absorption at $\lambda=250 \mathrm{~nm}$ can be ascribed to it.

\section{CONCLUSIONS}

Flash photolysis of the aliphatic ketone polymers PMVK, PBVK, and PMIK and 3,3-dimethyl-2-butanone at $\lambda_{\text {inc }}=265 \mathrm{~nm}$ showed that radicals were formed during the $15 \mathrm{~ns}$ flash. In the cases of PBVK and PMIK, acyl radicals, generated by the $\alpha$-scission, were detected by the characteristic absorption spectrum. Indication for the formation of polymeric acyl radicals $(\mathrm{R}-\mathrm{C}=\mathrm{O})$ was obtained for PBVK. Because the transient absorption spectrum observed at the end of the flash differed from the spectra observed with the other two polymers, the triplet biradical for PMVK was postulated as a transient with a lifetime of about $40 \mathrm{~ns}$.

It is noteworthy that a rather short triplet lifetime of $c a .6 \mathrm{~ns}$ has been estimated by Dan et $a .^{20}$ for PMVK on the basis of data from quenching experiments. Our findings that radicals are formed rapidly during the $15 \mathrm{~ns}$ flash, corroborate this earlier conclusion. Triplet lifetimes of a few ns were assumed for the other two polymers, PBVK and PMIK. Indications for the existence of triplets could not be obtained in this work from emission studies.

Acknowledgment. The authors are grateful to Dr. G. Beck for maintaining the Nd-laser and Mrs. U. Fehrmann and Mr. K. Imamura for synthesis of the polymers.

\section{REFERENCES}

1. G. Beck, G. Dobrowolski, J. Kiwi, and W. Schnabel, Macromolecules, 8, 9 (1975); J. Kiwi and W. Schnabel, ibid., 8, 430 (1975); W. Schnabel, Pure Appl. Chem., 51, 2373 (1979).

2. J. Faure, J.-P. Fouassier, D. J. Lougnot, and R. Salvin, Eur. Polym. J., 13, 891 (1977), J. Photochem., 5, 13 (1976).

3. M. V. Encinas and J. C. Scaiano, J. Chem. Soc., Perkin Trans. 2, 56 (1980); J. Am. Chem. Soc., 100, 7108 (1978); R. D. Small and J. C. Scaiano, Chem. Phys. Lett., 48, 354 (1977), 50, 431 (1977); 59, 246 (1978); J. Phys. Chem., 81, 2126 (1977).

4. I. Naito, Bull. Chem. Soc. Jpn., 56, 2851 (1983).

5. W. Schnabel, "Application of the Light Scattering Detection Method to Problems of Polymer Degradation, in Developments in Polymer Degradation 2," N. Grassie, Ed., Applied Science, London, 1979.

6. I. Naito, K. Koga, A. Kinoshita, and W. Schnabel, Eur. Polym. J., 16, 109 (1980).

7. I. Naito, R. Kuhlmann, and W. Schnabel, Polymer, 20, 165 (1979).

8. W. Schnabel, Makromol. Chem., 180, 1487 (1979).

9. J. E. Guillet and R. G. Norrish, Proc. R. Soc., Ser. A, 233, 153 (1955).

10. I. Naito, K. Imamura, H. Shintomi, K. Okamura, and A. Kinoshita, Polym. Photochem., 4, 149 (1984).

11. K. F. Wissbrum, J. Am. Chem. Soc., 81, 58 (1959).

12. A. R. Schultz, J. Polym. Sci., 47, 267 (1960).

13. A. Kinoshita, I. Naito, and S. Yamanaka, Bull. Tech. Assoc. Graphic Arts Jpn., 16, 109 (1977).

14. D. Lindenau, S. W. Beaven, and W. Schnabel, Eur. Polym. J., 13, 819 (1977).

15. A. C. Somersall and J. E. Guillet, Macromolecules, 5, 410 (1972).

16. S. Noda, K. Fueki, and Z. Kuri, J. Chem. Phys., 49, 3287 (1968).

17. N. C. Yang and E. D. Fiet, J. Am. Chem. Soc., 90, 504 (1968). 


\section{NAITo and W. SchNaBEL}

18. B. Blank, A. Henne, and H. Fischer, Helv. Chim. Acta, 57, 920 (1974).

19. I. Naito and W. Schnabel, Bull. Chem. Soc. Jpn., in press.

20. E. Dan, A. C. Sommersall, and J. E. Guillet, Macromolecules, 6, 228 (1973). 